\title{
Production planning of fish processed product under uncertainty
}

\author{
Herman Mawengkang ${ }^{1}$
}

(Received 14 March 2010; revised 17 September 2010)

\begin{abstract}
Marine fisheries play an important role in the economic development of Indonesia. Besides being the most affordable source of animal protein in the diet of most people in the country, this industrial sector could provide employment to thousands who live at coastal area. We consider the management of small scale traditional business at North Sumatera Province which processes fish into several local seafood products. The inherent uncertainty of data (for example, demand and fish availability), together with the sequential evolution of data over time leads the production planning problem to be a linear mixed-integer stochastic program. We use a scenario generation based approach for solving the model. The result shows the amount of each fish processed product and the number of workforce needed in each horizon planning.
\end{abstract}

http://anziamj . austms.org.au/ojs/index.php/ANZIAMJ/article/view/3020 gives this article, (c) Austral. Mathematical Soc. 2010. Published November 2, 2010. ISSN 1446-8735. (Print two pages per sheet of paper.) Copies of this article must not be made otherwise available on the internet; instead link directly to this URL for this article. 


\section{Contents}

1 Introduction

C785

2 Framework of two stage recourse model

C787

3 Problem background

C788

4 The stochastic programming model

C790

5 The solution basic approach

C791

6 The algorithm

C795

7 Computational results

C797

8 Conclusions

C798

References

C802

\section{Introduction}

Marine fisheries play an important role in the economic development of Indonesia. This industry could provide employment to people who live at coastal areas, besides being a primary source of animal protein. The fisheries industrial sector can be classified into three different parts: open sea fishing, fish cultivation and processing fish. This article focuses on the latter sector. Generally the processed fish industry in Indonesia is found near the coast. There are a lot of varieties of processed fish produced, such as smoked fish and salted fish. The management of the fish processing industry is dominated by local traditional businesses, using conventional management strategies. Consequently they do not have enough information regarding market demand 
and price. In this production planning situation, the current information may be certain, but future events are inevitably stochastic.

The proposed model explicitly permits the incorporation of uncertain parameters. Most of the references concerning optimisation problems in the presence of uncertainty come under the heading of stochastic programming. Birge [1] gives a good presentation of the basics. Two stage stochastic programs with recourse typify a particularly important class of models. In such models the objective function commonly corresponds to the minimisation of expected costs (linear or nonlinear), although it can also refer to the expected value of the absolute or quadratic deviations of certain specific goals or the variance of the second stage recourse function. Two kinds of decision variables exist. Those determined before the random variables have been revealed are called first stage or here-and-now decision variables and represent proactive decisions; they correspond to the production cost and workforce of the first period. Those determined after the realisation of the random variables are called second stage or recourse decision variables and represent reactive decisions made in recourse or response to the uncertainty factor. Mulvey et al. [4], Sen and Higle [6], and Rico Ramirez [5], for example, give more details.

In the case of discrete random variables, the resulting two stage recourse models are usually large and complex, and thus must be solved numerically using suitable algorithmic strategies. Most of these algorithms apply decomposition strategies that break the model down by scenario or stage in an iterative scheme, allowing the resolution of smaller models (smaller in comparison to the deterministic equivalent model in its extensive form which gave rise to the original two stage model). In this article, a feasible neighbourhood search method is proposed to solve an extended deterministic equivalent model, in which each first stage variable is replicated for each scenario, with an imposed equality for the new variable values (non-anticipativity constraints). Section 2 briefly reviews the two stage recourse model. Section 3 presents the problem background. The stochastic programming model of the problem is the subject of section 4. Section 5 presents the solution basic approach. Sections 7 and 8 present computational results and conclusions, respectively. 


\section{Framework of two stage recourse model}

This section briefly describes the framework of a two stage, stochastic, integer programming model. Van der Vlerk and Haneveld [7] give more details. The stochastic linear programming model is

$$
\begin{aligned}
& \min \vec{c}_{\chi}^{\top} \vec{x}+\sum_{s=1}^{s} p_{s}\left(\vec{q}^{\top} \vec{y}^{s}\right), \\
& \text { such that } A \vec{x}=\vec{b}, \\
& T^{s} \vec{x}+W \vec{y}^{s}=\vec{h}^{s}, \quad s=1, \ldots, S, \\
& \vec{x}, \vec{y}^{s} \geqslant 0, \quad s=1, \ldots, S .
\end{aligned}
$$

Equations (2) represent the first stage model and Equations (3) represent the second stage model. $\vec{x}$ is the vector of first stage decision variables which is scenario independent. The optimal value of $x$ is not conditional on the realisation of the uncertain parameters. $\overrightarrow{\mathcal{c}}_{x}$ is the vector of cost coefficient at the first stage. $A$ is the first stage coefficient matrix and $\vec{b}$ is the corresponding right-hand side vector. $\vec{y}^{s}$ is the vector of second stage (recourse) decision variables. $\vec{q}$ is the vector of cost (recourse) coefficient matrix and $\vec{h}^{s}$ is the corresponding right-hand-side vector and $\mathrm{T}^{s}$ is the matrix that ties the two stages together where $s \in \Omega$ represents scenarios in future and $p_{s}$ is the probability that scenario $s$ occurs. In the second stage model, the random constraint defined in (3), $\overrightarrow{\mathrm{h}}^{s}-\mathrm{T}^{s} \overrightarrow{\mathrm{x}}$, is the goal constraint: violations of this are allowed, but the associated penalty cost $\vec{q}^{\top} \vec{y}^{s}$ influences the choice of $\vec{x} . \vec{q}^{\top} \vec{y}^{s}$ is the recourse penalty cost or second stage value function and $\sum_{s=1}^{S} p_{s}\left(\vec{q}^{\top} \vec{y}^{s}\right)$ denotes the expected value of recourse penalty cost (second stage value function). 


\section{Problem background}

Fish and its processed products are the most affordable source of animal protein in the diet of most people. In Indonesia, most of the fish processing industries are found near the coast. In these industries fish are processed traditionally. There are eight kinds of fish product produced by the community: dried fish, salted fish, BBQ fish, pindang fish, smoked fish, fish preserved, pressed fish, and fish bowl.

The fish processing industry under investigation is located at the eastern coastal area of North Sumatra province of Indonesia. The industry has to make a production plan for these eight fish processed products to fulfil market demand over each period of time $t, t=1, \ldots, T$. In this case each period equals three months. Therefore there are four periods in a year.

Model parameter and decision variables used throughout this article are defined as follows.

\section{Sets}

- $\mathrm{T}=$ number of periods;

- $\mathrm{N}=$ set of products;

- $M=$ set of sources;

- $\mathrm{S}=$ set of scenarios.

\section{Variables}

- $x_{j t}=$ Quantity of product $j \in N$ in period $t \in T$ (units)

$-j=1$ for dried fish;

$-j=2$ for salted fish; 
$-j=3$ for BBQ fish;

$-j=4$ for pindang fish;

$-j=5$ for smoked fish;

$-j=6$ for fish preserved;

$-j=7$ for pressed fish;

$-j=8$ for fish bowl.

- $\mathfrak{u}_{\mathrm{it}}=$ Additional amount of resource $i \in M$ to purchase in $t \in T$ (unit).

- $k_{\mathrm{t}}=$ Number of workers required in period $t \in T$ (man-period).

- $k_{\mathrm{t}}^{-}=$Number of workers laid-off in period $\mathrm{t} \in \mathrm{T}$ (man-period).

- $\mathrm{k}_{\mathrm{t}}^{+}=$Number of additional workers in period $\mathrm{t} \in \mathrm{T}$ (man-period).

- $\mathrm{I}_{\mathrm{jt}}=$ Quantity of product $\mathrm{j} \in \mathrm{N}$ to be stored in period $\mathrm{t} \in \mathrm{T}$ (units).

- $\mathrm{B}_{\mathrm{jt}}=$ Under-fulfilment of product $j \in \mathrm{N}$ in period $\mathrm{t} \in \mathrm{T}$ (units).

\section{Parameters}

- $\alpha, \beta, \gamma, \delta, \mu, \rho, \lambda$ are all costs (in IDR, Indonesian currency, Rupiah).

- $D_{j t}=$ Demand for product $j \in N$ in period $t \in T$ (units).

- $\mathrm{u}_{\mathrm{jt}}=$ Upper bound on $\mathfrak{u}_{\mathrm{jt}}$.

- $r_{i j}=$ Amount of resource $i \in M$ needed to produce one unit of product $j \in N$.

- $f_{i t}=$ Amount of resource $i \in M$ available at time $t \in T$ (units).

- $a_{i j}=$ Number of worker needed to produce one unit of product $j \in N$. 


\section{The stochastic programming model}

Minimize

$$
\begin{aligned}
& \sum_{\mathrm{t} \in \mathrm{T}} \sum_{j \in N} \alpha_{j t} x_{j t}+\sum_{i \in M} \sum_{t \in T} \beta_{i t} u_{i t}+\sum_{t \in T} \mu_{t} k_{t}+\sum_{t \in T} \gamma_{t} k_{t}^{-} \\
& +\sum_{\mathrm{t} \in \mathrm{T}} \delta_{\mathrm{t}} k_{\mathrm{t}}^{+}+\sum_{s \in S} p_{s} \sum_{j \in N} \sum_{\mathrm{t} \in \mathrm{T}} \rho_{j \mathrm{t}}^{s} \mathrm{I}_{\mathrm{jt}}^{s}+\sum_{s \in S} p_{s} \sum_{j \in N} \sum_{\mathrm{t} \in \mathrm{T}} \lambda_{j \mathrm{t}}^{s} B_{j \mathrm{t}}^{s}
\end{aligned}
$$

subject to

$$
\begin{aligned}
& \sum_{j \in N} r_{j i} x_{j t} \leqslant f_{i t}+u_{i t}, \quad \text { for all } i \in M, t \in T, \\
& u_{i t} \leqslant u_{i t}, \quad \text { for all } i \in M, t \in T, \\
& \sum_{j \in N} a_{j} x_{j t} \leqslant k_{t}, \quad \text { for all } t \in T, \\
& k_{t}=k_{t-1}+k_{t}^{+}-k_{t}^{-}, \quad t=2, \ldots, T, \\
& x_{j t}+B_{j, t-1}^{s}+I_{j t}^{s}-B_{j t}^{s}=D_{j t}^{s}, \\
& \quad \text { for all } j \in N, t \in T \text { and } s \in S, \\
& x_{j t}, u_{i t}, k_{t}, k_{t}^{-}, k_{t}^{+}, I_{j t}^{s}, B_{j t}^{s} \geqslant 0, \\
& \quad \text { for all } j \in N, i \in M, t \in T \text { and } s \in S .
\end{aligned}
$$

In this production planning problem we decide: the quantity of each fish processed product to be produced in each period; the additional resource to be used; the number of regular additional and laying-off workers in each period.

The demand for each period is uncertain. Under the random demand in each period we should decide the number of each product to be stored in inventory or used to fulfil the under fulfilment for each period.

All of these decisions are formulated in expression (5) of the model as an objective function. Constraint (6) expresses that the amount of resource $i \in M$ 
needed to produce product $j \in N$ at least should have the same amount of resources available at time $t \in \mathrm{T}$ together with the additional resource needed. However, the additional resource needed has an upper bound, expression (7). In constraint (8), we have the number of workers needed to produce one unit product $j \in \mathbf{N}$. Constraint (9) ensures that the available workers in any period equals the number of worker from the previous period plus any change in the number of worker level during the current period. The change in the number of worker level may be due to either adding extra workers or laying off redundant workers. Constraint (10) determines either the quantity of product to be stored in inventory or to purchase from outside to fulfil the shortfall in meeting market demand.

The model formulated in expression (5) through to (11) is in deterministic equivalent form: the random factors are represented by scenarios; and in the objective function the corresponding random terms are premultiplied by the corresponding probabilities $p_{s}$. The method for transforming a stochastic programming model to its deterministic equivalent model was addressed by Mawengkang et al. [2].

\section{The solution basic approach}

Consider a mixed integer linear programming problem in the following form

$$
\min _{\vec{x}}\left\{\vec{c}^{\top} \vec{x}: A \vec{x} \leqslant \vec{b}, \vec{x} \geqslant 0\right\}
$$

with $x_{j}$ integer for some $j \in J$, where $J$ is an index set.

A component of the optimal basic feasible vector $\left(\vec{x}_{B}\right)_{k}$, solved as continuous, can be written as

$$
\left(\vec{x}_{B}\right)_{k}=\beta_{k}-\alpha_{k 1}\left(\vec{x}_{N}\right)_{1}-\cdots-\alpha_{k j}\left(\vec{x}_{N}\right)_{j}-\cdots-\alpha_{k, n-m}\left(\vec{x}_{N}\right)_{n-m} .
$$

Note that this expression can be found in the final tableau of the Simplex procedure. If $\left(\vec{x}_{B}\right)_{k}$ is an integer variable and we assume that $\beta_{k}$ is not an 
integer, the partitioning of $\beta_{k}$ into the integer and fractional components is

$$
\beta_{k}=\left[\beta_{k}\right]+f_{k}, \quad 0 \leqslant f_{k} \leqslant 1 .
$$

Suppose we wish to increase $\left(\vec{x}_{B}\right)_{k}$ to its nearest integer, $([\beta]+1)$. Based on the idea of suboptimal solutions we may elevate a particular nonbasic variable, say $\left(\vec{x}_{N}\right) j^{*}$, above its bound of zero, provided $\alpha_{k j *}$, as one of the element of the vector $\vec{\alpha}_{j *}$, is negative. Let $\Delta_{j *}$ be amount of movement of the non basic variable $\left(\vec{x}_{N}\right)_{j *}$, such that the numerical value of scalar $\left(\vec{x}_{B}\right)_{k}$ is integer. Referring to Equation (13), $\Delta_{j *}$ can then be

$$
\Delta_{f^{*}}=\frac{1-f_{k}}{-\alpha_{k j *}}
$$

while the remaining nonbasic stays at zero. After substituting (15) into (13) for $(x N)_{j *}$ and taking into account the partitioning of $\beta_{k}$ given in (14), we obtain $\left(\vec{x}_{B}\right)_{k}=[\beta]+1$. Thus, $\left(\vec{x}_{B}\right)_{k}$ is now an integer. Clearly, a nonbasic variable plays an important role to integerize the corresponding basic variable. Therefore, the following result is necessary in order to confirm that there must be a non-integer variable to work with in the integerizing process

Theorem 1 Suppose the problem in Equation (12) has an optimal solution, then some of the nonbasic variables $\left(\vec{x}_{N}\right)_{j}, j=1, \ldots, n$, must be non-integer variables.

Proof: Solve the problem using continuous of slack variables (which are non-integer, except in the case of equality constraint). If we assume that the vector of basic variables $\vec{x}_{B}$ consists of all the slack variables then all integer variables would be in the nonbasic vector $\vec{x}_{N}$ and therefore integer valued.

The other components, $\left(\vec{x}_{B}\right)_{i \neq k}$, of vector $\left(\vec{x}_{B}\right)$ will also be affected as the numerical value of the scalar $\left(\vec{x}_{N}\right)_{j *}$ increases to $\Delta_{j} *$. Consequently, if some element of vector $\alpha_{j *}$, that is, $\alpha_{j *}$ for $i \neq k$, are positive, then the corresponding element of $\vec{x}_{B}$ will decrease, and eventually may pass through zero. 
Due to the non-negativity restriction a minimum ratio test is needed in order to see what is the maximum movement of the nonbasic $\left(\vec{x}_{N}\right)_{j *}$ such that all components of $\vec{x}$ remain feasible. This ratio test includes two cases:

1. a basic variable $\left(\vec{x}_{B}\right)_{i \neq k}$ decreases to zero (lower bound) first;

2. the basic variable $\left(\vec{x}_{B}\right)_{k}$ increases to an integer.

Specifically, corresponding to each of these two cases above, one would compute

$$
\theta_{1}=\min _{i \neq k \mid \alpha_{j *}>0}\left[\frac{\beta_{i}}{\alpha_{j *}}\right] \text { and } \theta_{2}=\Delta_{j *} .
$$

How far one can release the nonbasic $\left(\vec{x}_{N}\right)_{j *}$ from its bound of zero, such that vector $\vec{x}$ remains feasible, depends on the ratio test

$$
\theta^{*}=\min \left(\theta_{1}, \theta_{2}\right) \text {. }
$$

If $\theta^{*}=\theta_{1}$, one of the basic variable $\left(\vec{x}_{B}\right), i \neq k$, will hit the lower bound before $\left(\vec{x}_{B}\right)_{k}$ becomes integer. If $\theta^{*}=\theta_{2}$, the numerical value of the basic variable $\left(\vec{x}_{B}\right)_{k}$ will be integer and feasibility is still maintained. Analogously, we would be able to reduce the numerical value of the basic variable $\left(\vec{x}_{B}\right)_{k}$ to its closest integer $\left[\beta_{k}\right]$. Consider the movement of a particular nonbasic variable, $\Delta$, as expressed in Equation (15). The only factor that one needs to calculate is the corresponding element of vector $\vec{\alpha}$. A vector $\vec{\alpha}_{j}$ can be expressed as $\vec{\alpha}_{j}=B^{-1} \vec{a}_{j}, j=1, \ldots, n-m$. Therefore, in order to get a particular element of vector $\vec{\alpha}_{j}$ we should be able to distinguish the corresponding column of matrix $[\mathrm{B}]^{-1}$. Suppose we need the value of element $\vec{\alpha}_{k j *}$, letting $\vec{v}_{k}^{\top}$ be the kth column vector of $[\mathrm{B}]^{-1}$, we then have

$$
\vec{v}_{\mathrm{k}}^{\top}=\vec{e}_{\mathrm{k}}^{\top} \mathrm{B}^{-1} \text {, }
$$

subsequently, the numerical value of

$$
\alpha_{k j *}=v_{k}^{\top} B^{-1} \text {. }
$$

In Linear Programming terminology the operation conducted in Equations (17) and (18) is called the pricing operation. The vector of reduced costs $\vec{d}_{j}$ is 
used to measure the deterioration of the objective function value caused by releasing a nonbasic variable from its bound. Consequently, in deciding which nonbasic should be released in the integerizing process, the vector $\vec{d}_{j}$ must be taken into account such that deterioration is minimized. Recall that the minimum continuous solution provides a lower bound to any integer feasible solution. Nevertheless, the amount of movement of particular nonbasic variable as given in Equation (15) depends in some way on the corresponding element of vector $\vec{\alpha}_{j}$. Therefore, the deterioration of the objective function value due to releasing a nonbasic variable $\left(\vec{x}_{N}\right)_{j *}$ so as to integerize a basic variable $\left(\vec{x}_{B}\right)_{k}$ may be measured by the ratio $\left|d_{k} / \alpha_{k j *}\right|$ where $|a|$ means the absolute value of scalar $a$. In order to minimize the deterioration of the optimal continuous solution we then use the following strategy for deciding which nonbasic variable may be increased from its bound of zero, that is,

$$
\min _{j}\left\{\left|\frac{\vec{d}_{k}}{\vec{\alpha}_{k j *}}\right|\right\}, \quad j=1, \ldots, n-m .
$$

From the 'active constraint' strategy and the partitioning of the constraints corresponding to basic (B), superbasic $(S)$ and nonbasic $(N)$ variables we write

$$
\begin{aligned}
& \left(\begin{array}{ccc}
\mathrm{B} & \mathrm{S} & \mathrm{N} \\
& & \mathrm{I}
\end{array}\right)\left(\begin{array}{c}
\vec{x}_{B} \\
\vec{x}_{S} \\
\vec{x}_{N}
\end{array}\right)=\left(\begin{array}{c}
\vec{b} \\
\vec{b}_{N}
\end{array}\right) \\
& B \vec{x}_{B}+S \vec{x}_{S}+N \vec{x}_{N}=\vec{b} \text { and } \vec{x}_{N}=\vec{b}_{N} .
\end{aligned}
$$

The basis matrix B is assumed to be square and nonsingular, we get

$$
\vec{\chi}_{B}=\vec{\beta}-W \vec{x}_{s}-\alpha \vec{x}_{N}
$$

with $\vec{\beta}=\mathrm{B}^{-1} \overrightarrow{\mathrm{b}}, \mathrm{W}=\mathrm{B}^{-1} \mathrm{~S}, \alpha=\mathrm{B}^{-1} \mathrm{~N}$.

Expression (21) indicates that the nonbasic variables are being held equal to their bound. It is evident through the 'nearly' basic expression of Equation (22), the integerizing strategy discussed previously, designed for the 
problem can be implemented. Particularly, we would be able to release a nonbasic variable from its bound, Equation (21), and exchange it with a corresponding basic variable in the integerizing process, although the solution would be degenerate.

Currently, we are in a position where a particular basic variable, $\left(\vec{x}_{B}\right)_{k}$ is being integerized, thereby a corresponding nonbasic variable, $\left(\vec{x}_{N}\right)_{j *}$, is being released from its bound of zero. Suppose the maximum movement of $\left(\vec{x}_{N}\right)_{j *}$ satisfies $\theta^{*}=\Delta_{j *}$ such that $\left(\vec{x}_{B}\right)_{k}$ is integer valued to exploit the manner of changing the basis, we would be able to move $\left(\vec{x}_{N}\right)_{j *}$ into B (to replace $\left(\vec{x}_{B}\right)_{k}$ ) and integer-valued $\left(\vec{x}_{B}\right)_{k}$ into $S$ in order to maintain the integer solution. We now have a degenerate solution since a basic variable is at its bound. The integerizing process continues with a new set $[B, S]$. In this case, eventually we end up with all of the integer variables being superbasic.

\section{The algorithm}

After solving the relaxed problem, the procedure for searching for a suboptimal but integer feasible solution from an optimal continuous solution is as follows. Let $x=[x]+f, 0 \leqslant f \leqslant 1$, be the (continuous) solution of the relaxed problem, $[x]$ is the integer component of non-integer variable $x$ and $f$ is the fractional component.

1. Get row $i^{*}$ the smallest integer infeasibility, such that $\delta_{i *}=\min \left(f_{i}, 1-\right.$ $\left.f_{i}\right)$.

2. Calculate $v_{i *}^{\top}=e_{i *}^{\top} B^{-1}$ (a pricing operation)

3. Calculate $\sigma_{i j}=v_{i *}^{\top} a_{j}$ with $j=\operatorname{argmin}_{j}\left\{\left|d_{j} / \sigma_{i j}\right|\right\}$

(a) For nonbasic $j$ at lower bound:

- if $\sigma_{i j}<0$ and $\delta_{i *}=f_{i}$ calculate $\Delta=\left(1-\delta_{i *}\right) /-\sigma_{i j}$; 
- if $\sigma_{i j}>0$ and $\delta_{i *}=1-f_{i}$ calculate $\Delta=\left(1-\delta_{i *}\right) / \sigma_{i j}$;

- if $\sigma_{i j}<0$ and $\delta_{i *}=1-f_{i}$ calculate $\Delta=\delta_{i *} /-\sigma_{i j}$.

(b) For nonbasic $j$ at upper bound:

- if $\sigma_{i j}<0$ and $\delta_{i *}=1-f_{i}$ calculate $\Delta=\left(1-\delta_{i *}\right) /-\sigma_{i j}$;

- if $\sigma_{i j}>0$ and $\delta_{i *}=f_{i}$ calculate $\Delta=\left(1-\delta_{i *}\right) / \sigma_{i j}$;

- if $\sigma_{i j}>0$ and $\delta_{i *}=1-f_{i}$ calculate $\Delta=\mathrm{d} \delta_{i *} / \sigma_{i j}$;

- if $\sigma_{i j}<0$ and $\delta_{i *}=f_{i}$ calculate $\Delta=\delta_{i *} /-\sigma_{i j}$.

(c) Otherwise go to next non-integer, nonbasic or superbasic $\mathbf{j}$ (if available). Eventually the column $j^{*}$ is to be increased from LB or decreased from UB. If none, go to next $i^{*}$.

4. Calculate $\alpha_{j *}=B^{-1} \alpha_{j *}$, that is, solve $B \alpha_{j *}=\alpha_{j *}$ for $\alpha_{j *}$.

5. Ratio test: there would be three possibilities for the basic variables in order to stay feasible due to the releasing of nonbasic $j^{*}$ from its bounds.

- If $\boldsymbol{j}^{*}$ lower bound, let

$$
A=\min _{i^{\prime} \neq i^{*} \mid \alpha_{i j *}>0}\left[\frac{x_{B_{i^{\prime}}}-l_{i^{\prime}}}{\alpha_{i j^{*}}}\right], \quad B=\min _{i^{\prime} \neq i^{*} \mid \alpha_{i j *}<0}\left[\frac{u_{i^{\prime}}-x_{B_{i}^{\prime}}}{-\alpha_{i j^{*}}}\right],
$$

and $C=\Delta$. Then the maximum movement of $j^{*}$ depends on $\theta^{*}=\min (A, B, C)$

- If $\boldsymbol{j}^{*}$ upper bound, let

$$
A^{\prime}=\min _{i^{\prime} \neq i^{*} \mid \alpha_{i j *}<0}\left[\frac{x_{B_{i^{\prime}}}-l_{i^{\prime}}}{\alpha_{i j^{*}}}\right], \quad B^{\prime}=\min _{i^{\prime} \neq i^{*} \mid \alpha_{i j *}>0}\left[\frac{u_{i^{\prime}}-x_{B_{i}^{\prime}}}{-\alpha_{i j^{*}}}\right],
$$

and $C^{\prime}=\Delta$. Then the maximum movement of $j^{*}$ depends on $\theta^{*}=\min \left(A^{\prime}, B^{\prime}, C^{\prime}\right)$.

6. Exchanging basis for the three possibilities. 
(a) If $A$ or $A^{\prime}$,

- $x_{B_{i^{\prime}}}$ becomes nonbasic at lower bound $l_{i^{\prime}}$,

- $x_{j^{*}}$ becomes basic (replaces $x_{B_{i^{\prime}}}$ ),

- $x_{i^{*}}$ stays basic (non-integer).

(b) If $\mathrm{B}$ or $\mathrm{B}^{\prime}$,

- $x_{B_{i^{\prime}}}$ becomes nonbasic at upper bound $\mathfrak{u}_{i^{\prime}}$,

- $\boldsymbol{x}_{j^{*}}$ becomes basic (replaces ${x_{B_{i^{\prime}}}}$ ),

- $x_{i^{*}}$ stays basic (non-integer).

(c) If $\mathrm{C}$ or $\mathrm{C}^{\prime}$,

- $x_{j^{*}}$ becomes basic (replaces $\left.x_{i}^{*}\right)$,

- $x_{i^{*}}$ becomes superbasic at integer valued.

7. Repeat from step 1.

\section{Computational results}

The planning horizon covers every three months, that is, $\mathrm{T}=\{1,2,3,4\}$. After we surveyed the locations, we found out that the market situation for the eight fish processed products could fit within three possible situations, good, fair and poor, with associated probabilities of $0.30,0.50$ and 0.20 respectively. Nevertheless, the method addressed by Mawengkang and Suherman [3] could be used in order to get an efficient number of scenarios. The data of the problem are described in Table 1 through to Table 8.

Data for production cost for each fish processed product, cost for holding products in inventory in each period and the cost to purchase from outside in order to meet the demand are shown in Table 2. Table 3 presents the cost 


\begin{tabular}{crrr} 
TABLE 1: & Upper bound for additional resources. \\
\hline Period & Machine 1 & Machine 2 & Machine 3 \\
\hline 1 & 300 & 300 & 200 \\
2 & 300 & 300 & 200 \\
3 & 250 & 300 & 200 \\
4 & 200 & 250 & 250 \\
\hline
\end{tabular}

incurred for additional resources and capacity of resource in each machine. The upper bound for additional resources can be found in Table 1.

The workforce needed to produce each fish product for the whole period is given in Table 5 . Table 4 gives the cost for hiring workforce. Table 6 shows resources needed to produce each fish product in each source (machine). The computational results, presented in Table 8, describes the quantity of each product to be produced. Additional resources needed, and the plan for workforce are given in Table 7.

\section{Conclusions}

This article developed a two stage recourse model for the production planning problem of a fish processing industry in a coastal area with stochastic demand. The model is adequate for solving the planning problem faced by the management of the industry. The model estimates the number of workers which is very useful for the industry so they will be able to schedule the local people. We also propose an algorithm for solving the mixed integer stochastic programming problem. 


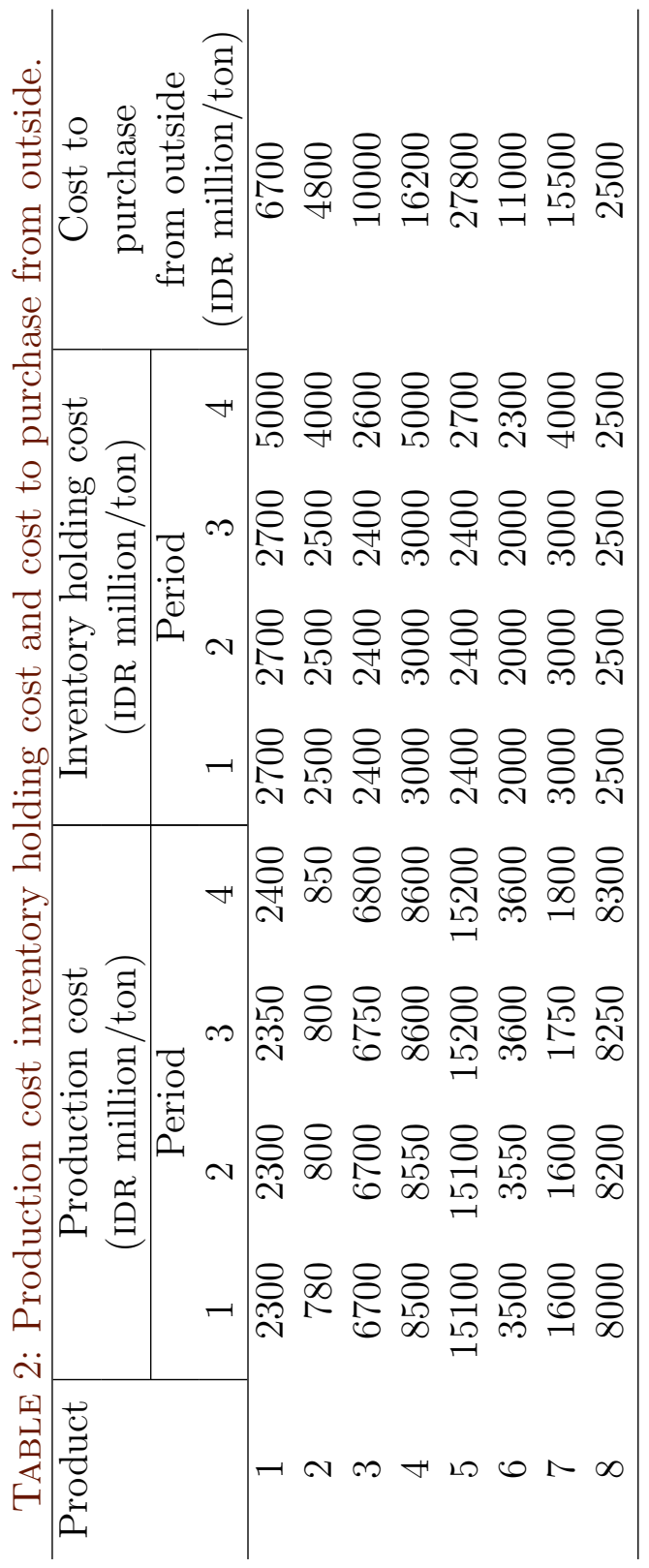


TABLE 3: Additional resource cost and capacity of resource.

\begin{tabular}{c|cccc|cccc}
\hline Sources & \multicolumn{4}{|c|}{$\begin{array}{c}\text { Additional resource cost } \\
\text { (IDR/ton) }\end{array}$} & \multicolumn{4}{c}{ Capacity of resource available } \\
& \multicolumn{4}{c}{ Period } & \multicolumn{4}{c}{ Period } \\
\cline { 2 - 9 } & 1 & 2 & 3 & 4 & 1 & 2 & 3 & 4 \\
\hline M1 & 45600 & 45800 & 45800 & 45900 & 20000 & 20000 & 20000 & 19000 \\
M2 & 34300 & 34600 & 34600 & 34700 & 18000 & 18000 & 19000 & 17000 \\
M3 & 32200 & 32300 & 32300 & 32500 & 21000 & 20000 & 21000 & 20000 \\
\hline
\end{tabular}

TABLE 4: Cost for workforce (IDR million/man-period).

\begin{tabular}{ccccc}
\hline Cost notation & \multicolumn{4}{c}{ Period } \\
& 1 & 2 & 3 & 4 \\
\hline$\mu$ & 22000 & 22500 & 22500 & 23000 \\
$\gamma$ & 24000 & 24000 & 25500 & 26000 \\
$\delta$ & 25000 & 25000 & 25600 & 27000 \\
\hline
\end{tabular}

TABLE 5: Workforce needed to produce each product. $\begin{array}{lllllllll}\text { Product } & 1 & 2 & 3 & 4 & 5 & 6 & 7 & 8\end{array}$

\begin{tabular}{lllllllll} 
Work force (man/ton) & 6 & 12 & 24 & 24 & 24 & 20 & 15 & 8 \\
\hline
\end{tabular}

TABLE 6: Resources needed for each product (ton). Sources

\begin{tabular}{lllllllll} 
& 1 & 2 & 3 & 4 & 5 & 6 & 7 & 8 \\
\hline Machine 1 & 6 & 5 & 6 & 8 & 7 & 6 & 5 & 9 \\
Machine 2 & 4 & 4 & 5 & 6 & 6 & 5 & 5 & 8 \\
Machine 3 & 5 & 3 & 5 & 6 & 6 & 5 & 5 & 7 \\
\hline
\end{tabular}


TABLE 7: Additional resource and workforce plan.

\begin{tabular}{c|ccc|ccc}
\hline Period & \multicolumn{2}{|c|}{$\begin{array}{c}\text { Additional resources } \\
\text { to be used (ton) }\end{array}$} & \multicolumn{3}{|c}{ Workforce plan } \\
\cline { 2 - 7 } & \multicolumn{2}{|c|}{$\begin{array}{c}\text { Resource machines } \\
1\end{array}$} & 2 & 3 & $\begin{array}{c}\text { Reg. } \\
\text { Policy } \\
\text { Add. }\end{array}$ & Lay off \\
& & & & 38 & 35 & 0 \\
\hline 1 & 12.20 & 9.80 & 8.65 & 35 & 0 & 0 \\
2 & 12.20 & 9.80 & 8.75 & 35 & 0 & 0 \\
3 & 12.20 & 9.70 & 8.65 & 35 & 0 & 0 \\
4 & 16.95 & 13.80 & 12.55 & 47 & 12 & \\
\hline
\end{tabular}

TABLE 8: The number of each product to be produced (ton).

\begin{tabular}{ccccc}
\hline Product & \multicolumn{4}{c}{ Period } \\
& 1 & 2 & 3 & 4 \\
\hline 1 & 25000 & 25000 & 36000 & 30000 \\
2 & 90000 & 90000 & 90000 & 95000 \\
3 & 20000 & 20000 & 20000 & 30000 \\
4 & 20000 & 20000 & 20000 & 30000 \\
5 & 20000 & 20000 & 20000 & 30000 \\
6 & 20000 & 20000 & 20000 & 30000 \\
7 & 20000 & 20000 & 20000 & 30000 \\
8 & 20000 & 20000 & 20000 & 30000 \\
\hline
\end{tabular}




\section{References}

[1] Birge J. R., Louveaux F. V. Introduction to stochastic programming. New York: Springer; 1997. C786

[2] Mawengkang H., Saib Suwilo, Opim S. Sitompul. Revision Modeling of Two-Stage Stochastic Programming Problem. Journal of Industrial System 2006; 7 (4):6-10. C791

[3] Mawengkang H., Suherman. A Heuristic Method of Scenario Generation in Multi-Stage Decision Problem under Uncertainty. Journal of Industrial System 2007; 8(2) : 98-105. C797

[4] Mulvey J. M., Van derbei R., Zenios S. Robust optimization of large scale systems. Operations research 1995;43 (2): 264-281. C786

[5] Rico-Ramirez V. Two-stage stochastic linear programming: a tutorial. SIAG/OPT Views-and-News 2002; 13 (1): 8-14. C786

[6] Sen S., Higle J. L. Introductory tutorial on stochastic linear programming models. Interfaces 1999; 29 (2): 33-61. C786

[7] Van der Vlerk M. H., Haneveld W. K. Stochastic integer programming: General models and algorithms. Annals of Operations Research,85:39-57,1999. C787

\section{Author address}

1. Herman Mawengkang, Department of Mathematics, The University of Sumatera Utara, INDONESIA.

mailto: hmawengkang@yahoo.com 